\title{
Series Solution of One-Dimensional Stationary Thermal Explosion Problems
}

Ahmed R. Shouman, A. Burl Donaldson, John Werth

Prepared by Sandia Laboratories. Albuquerque New Mexico 87115

and Livermore, California 94550 for the United States Energy Research

and Development Administration under Contract AT $(29-1) 789$

Printed June 1976

\section{Sandia Laboratories}

When printing a copy of any digitized SAND

Report, you are required to update the markings to current standards. 
Issued by Sandia Laboratories, operated for the United States Energy Research and Development Administration by Sandia Corporation.

\section{NOTICE}

This report was prepared as an account of work sponsored by the United States Government. Neither the United States nor the United States Energy Research and Development Administration, nor any of their employees, nor any of their contractors, subcontractors, or their employees, makes any warranty, express or implied, or assumes any legal liability or responsibility for the accuracy, completeness or usefulness of any information, apparatus, product or process disclosed, or represents that its use would not infringe privately owned rights.

Printed in the United States of America

Available from

National Technical Information Service

U. S. Department of Commerce

5285 Port Royal Road

Springfield, Virginia 22161

Price: Printed Copy $\$ 5.00$; Microfiche $\$ 2.25$ 
SAND76-0247

Unlimited Release

Printed June 1976

SERIES SOLUTION OF ONE-DIMENSIONAL STATIONARY

THERMAL EXPLOSION PROBLEMS

\author{
Ahmed R. Shouman \\ Professor of Mechanical Engineering \\ New Mexico State University \\ Las Cruces, New Mexico \\ A. Burl Donaldson \\ Detonating Components Div. 2513 \\ Sandia Laboratories \\ Albuquerque, New Mexico 87115 \\ and \\ John Werth \\ Assistant Professor of Mathematics \\ University of Nevada \\ Las Vegas, Nevada
}

\begin{abstract}
A series solution is obtained for one-dimensional, stationary thermal explosion problems by expressing the temperature in the reactive material in terms of an even power series of the position variable. Coefficients of this series are determined by replacing terms in the energy equation with their series equivalents and by equating coefficients of like powers. The resulting series is examined for the condition of incipient thermal explosion, and the resulting critical parameters are tabulated. Specific geometries treated are the slab and the solid cylinder and sphere.
\end{abstract}




\section{CONTENTS}

$\begin{array}{lr} & \text { Page } \\ \text { Nomenclature } & 6 \\ \text { Introduction } & 7 \\ \text { Analysis } & 8 \\ \text { Conclusions } & 15 \\ \text { References } & 16\end{array}$

TABLES

Table

Page

1 The a(1, n 's for Cartesian Geometry $(k=0) \quad 17$

2 The $a(1, n)$ 's for Cylindrical Geomerty $(\mathrm{k}=1) \quad 21$

3 The $a(1, n)$ 's for Spherical Geometry $(k=2) \quad 25$

4 Critical Parameters for Cartesian Geometry $(\mathbf{k}=0) \quad 29$

$5 \quad$ Critical Parameters for Cylindrical Geometry $(k=1) \quad 32$

6 Critical Parameters for Spherical Geometry $(k=2)$ 


\section{Nomenclature}

Variables
$a, b, c$
subscripted coefficients used to represent temperature profile
E
activation energy
K
thermal conductivity of reactant
$\mathbf{k}$
integer which specifies geometry
L
half-thickness of reactant
$\mathrm{n}$
index used in series
$\mathrm{p}$
index used in series
Q
heat of reaction
R
gas constant
$\mathrm{T}$
absolute temperature
$\mathbf{x}$
dimensional position
Z
pre-exponential (frequency factor)
$\delta$
critical parameter
$\theta$
nondimensional temperature ( $\equiv \mathrm{RT} / \mathrm{E})$
$\xi$
nondimensional position $\left[\equiv\left(\frac{\rho Q Z R}{\mathrm{KE}}\right)^{1 / 2} \times\right]$
$\rho$
density of reactant

Subscripts
m
maximum
$\mathbf{s}$
surface

Superscript

*

critical 


\title{
SERIES SOLUTION OF ONE-DIMENSIONAL STATIONARY \\ THERMAL EXPLOSION PROBLEMS
}

\author{
Introduction
}

In general, the reaction rate of exothermically (self-heating) decomposing materials increases as absolute temperature increases. As a result, an increase in the boundary temperature of a self-heating body results in a correlative increase in the internal heating rate. At some sufficiently high boundary temperature, the internal heat generation for a given body can marginally be conducted to and dissipated at the boundary. When the boundary temperature is raised above this point, accelerating self-heating and finally thermal explosion will occur within the body.

The boundary temperature at which thermal explosion occurs is a function of the characteristics of the reactive material and its dimension(s). Similarly, if a boundary temperature is specified for a given material and the dimension of the body is increased, a condition occurs in which any further increase in dimension leads to thermal explosion. This dimension for impending thermal explosion is a function of the characteristics of the reactive material and its boundary temperature(s). Hence, either critical thickness for a given boundary temperature or critical boundary temperature for a given thickness can specify the condition of incipient thermal explosion.

Investigation of the theory of thermal explosion began with the work of Semenov [1]. Semenov considered a reactive body to be of uniform temperature, and he determined the maximum value of this temperature as a function of dissipation at the boundary. Frank-Kamenetskii [2] considered the problem 
of a symmetric slab with a specified boundary temperature and internal temperature gradients. In his approach, an approximate form of the self-heating term (based on the characteristics of common explosives) was used to allow solution. By this method, a combination of thickness and boundary temperature was found to be invariant at the critical condition. Chambré [3] extended the FrankKamenetskii exponential approximation to the analysis of cylinders and spheres and found a similar invariance in Frank-Kamenetskii's critical condition for these geometries. More recent investigations $[4,5,6]$ have shown that the invariance is valid for large thickness (or small boundary temperature) but does not hold for all possible conditions.

The series solution method used in this study is similar to a method which has been widely applied to solving ordinary linear differential equations with variable coefficients, i.e., the method of Frobenius. The present approach differs from the series method of Reference 5 wherein the coefficients were evaluated from the boundary conditions and the energy-equation at internal location(s)。

\section{Analysis}

If the standard Arrhenius model is assumed for heat production, the dimensionless stationary energy equation and standard boundary conditions (B.C.) are

$$
\begin{array}{r}
\frac{d^{2} \theta}{d \xi^{2}}+\frac{k}{\xi} \frac{d \theta}{d \xi}=-\exp (-1 / \theta) \\
\text { B.C. } 1 . \frac{d \theta}{d \xi}(0)=0\left[\theta(0)=\theta_{m}\right]
\end{array}
$$




$$
\text { 2. } \theta(L)=\theta_{s}
$$

where

$$
\begin{aligned}
& k=0 \text { for slab geometry } \\
& k=1 \text { for cylinder geometry } \\
& k=2 \text { for sphere geometry }
\end{aligned}
$$

By the steady-state nature of Eq. (1), no depletion is presumed. This is of course an approximation because any self-heating is necessarily a result of reaction. However, at temperatures only moderately below the critical temperature, self-heating is negligible; and, at the critical temperature or above, thermal explosion results after only a small fraction of the material has been consumed. Hence, Eq. (1) is a good representation of a quasi-stationary process. Boundary Condition 1 is a result of symmetry about the mid-plane of a slab, the center of an infinite cylinder, or the center of a sphere. The temperature at this location is designated $\theta_{\mathrm{m}}$; although it is normally an unknown quantity, it will be used throughout the analysis and will subsequently be related to the boundary temperature $\theta_{s}$ at the outside surface $(\xi=L)$.

Because thermal explosion has been shown to be a result of an upward displacement of internal temperature for this type of problem [6] and because the reaction rate is highest at the highest temperature, $\hat{~}_{\mathrm{m}}$, a series of the form

$$
\theta=\theta_{m}-\sum_{n=1}^{\infty} a(1, n) \xi^{2 n}
$$


is suggested. Only even powers of $\xi$ are used for the purpose of satisfying the condition of symmetry about the origin. The series can be truncated at any integer $\mathrm{n}$; however, the accuracy of the final solution is improved as the number of terms increases.

Equation (2) is now substituted into the left side of Eq. (1) to give

$$
\frac{d^{2} \theta}{d \xi^{2}}+\frac{k}{\xi} \frac{d \theta}{d \xi}=\sum_{n=1}^{\infty}\left\{2 n[(2 n-1)+k] a(1, n) \xi^{2 n-2}\right\} .
$$

Inversion of Eq. (2) for the exponential term of the right side of Eq. (1) can be accomplished by the binomial expansion. The result is

$$
\frac{1}{\theta}=\frac{1}{\theta_{m}} \sum_{p=0}^{\infty} \theta_{m}^{-p}\left(\sum_{n=1}^{\infty} a(1, n) \xi^{2 n}\right)^{p} .
$$

Because $\theta_{\mathrm{s}}<\theta_{\mathrm{m}}$, the expansion is valid for all $\xi \leq \mathrm{L}$.

Substitution of Eqs. (3) and (4) into Eq. (1) yields

$$
\begin{aligned}
& \sum_{n=1}^{\infty}\left\{2 n[(2 n-1)+k] a(1, n) \xi^{2 n-2}\right\} \\
& \quad=\exp \left(-\frac{1}{\theta_{m}}\right) \cdot \prod_{p=1}^{\infty} \exp \left[\theta_{m}^{-(p+1)}\left(\sum_{n=1}^{\infty} a(1, n) \xi^{2 n}\right)\right] .
\end{aligned}
$$


Now, to complete the solution, the exponential can be expressed as a series. Then by equating coefficients of like powers of $\xi$ in Eq. (5), the a(1,n)'s can be determined and Eq. (2) then expresses the temperature profile. However, by hand this procedure is rather tedious for the higher terms in the series. $\mathrm{Be}-$ cause the series becomes more slowly convergent as the geometry changes from slab to cylinder to sphere, machine computation of the coefficients is required in order to obtain a sufficient number of terms. Thus, the following discussion will briefly outline an algorithm which was used for computer determination of the $a(1, n)$ 's.

For shorthand, define

$$
y=\sum_{n=1}^{\infty} a(1, n) \xi^{2 n}
$$

Then the product term in Eq. (5) can be written

$$
\prod_{p=1}^{\infty} \exp \left(-\frac{y^{p}}{\theta_{m}^{p+1}}\right)
$$

By writing the exponential as a series and using series multiplication, the alternate form

$$
\prod_{p=1}^{\infty} \exp \left(-\frac{y^{p}}{\theta_{m}^{p+1}}\right)=\sum_{j=0}^{\infty} b(r, j) y^{j}
$$


can be expressed. Here, the $b(r, j)$ 's represent the collection of coefficients from all of the $y^{j}$ terms. It can be shown that the $b(r, j)$ 's can be related to lower coefficients by

$$
b(r, j)=\sum_{\ell=0}^{\ell \leq \frac{j}{r}} \frac{(-1)^{\ell} b(r-1, j-r \ell)}{\ell ! \theta_{m}^{(r+1) \ell}} .
$$

Using the definition of $y$, the $y^{j_{1}} s$ can be expressed as

$$
y^{j}=\sum_{i=j}^{\infty} a(j, i) \xi^{2 i}
$$

It can then be shown that the $a(j, i)$ 's are related to lower order coefficients by the expression

$$
a(j, i)=\sum_{q=1}^{q=i+1+j} a(1, q) a(j-1, i-q)
$$

Now, substitution of Eq. (8) into Eq. (6) yields

$$
\prod_{p=1}^{\infty} \exp \left(-\frac{y^{p}}{\theta_{m}^{p+1}}\right)=b(r, o)+\sum_{n=1}^{\infty} c(n) \xi^{2 n},
$$

where

$$
c(n)=\sum_{m=1}^{n} b(n, m) a(n, m)
$$


Recognizing that $b(r, 0)=1$, Eqs. (10) and (5) can be combined to give

$$
\sum_{n=1}^{\infty} 2 n[(2 n-1)+k] a(1, n) \xi^{2 n-2}=1+\sum_{n=1}^{\infty} c(n) \xi^{2 n} .
$$

Finally, by equating like powers of $\xi$ in Eq. (11),

$$
a(1, n)=\frac{\exp \left(-1 / \theta_{m}\right)}{2 n[(2 n-1)+k]} c(n-1)
$$

The symbolic computer language Formac was used with the above equations to determine the $a(1, n)$ 's as functions of $\theta_{m}$ for $k=0,1,2$ and $n=1,2,3$, ... These are given in Tables 1 through 3 for the slab, cylinder, and sphere geometries, respectively.

For a self-heating material with a fixed boundary temperature, the criterion for thermal explosion has been shown to be [6]

$$
\left(\frac{\partial L}{\partial \theta_{m}}\right)_{\theta_{S}}=0
$$

That is, for a constant $\theta_{\mathbf{s}}$, at the onset of explosion an infinitesimal increase in length is accompanied by a large increase in $\theta_{\mathrm{m}}$. Mathematically, on a graph of $L$ as a function of $\theta_{m}$ for constant $\theta_{s}$, there is a region in which, for each $L$, three values of $\theta_{\mathrm{m}}$ are found; thermal explosion corresponds to the relative maxima $\left(L=L_{1}^{*}\right)$, and, although no physical interpretation has yet been shown, the relative minima is called the extinction limit $\left(L=L_{2}^{*}\right)$. To examine this criterion, Eq. (2) is evaluated at $\xi=L,\left(\theta=\theta_{s}\right)$, and the operation of Eq. (13) 
is taken. Thus,

$$
0=1-\sum_{n=1}^{\infty}\left[\frac{\partial a(1, n)}{\partial \theta_{m}}\right]_{\theta_{s}} L^{* 2 n},
$$

where the $L^{*}$ 's refer to the critical length for the onset of thermal explosion or extinction. By defining a critical delta based on $\theta_{\mathrm{m}}$,

$$
\delta_{m}=\frac{L^{* 2}}{\theta_{m}^{2} \exp \left(1 / \theta_{m}\right)} \text {, }
$$

Equation (14) can be written as

$$
0=1-\sum_{n=1}^{\infty} \theta_{m}^{2} \exp \left(1 / \theta_{m}\right)\left[\frac{\partial a(1, n)}{\partial \theta}\right]_{\theta_{s}} \delta_{m}^{n} .
$$

Equation (16) can be solved to give $\delta_{\mathrm{m}}$ as a function of $\theta_{\mathrm{m}}$. Then the boundary temperature corresponding to this critical condition is given by evaluating Eq. (2) at $L=L_{1}^{*}$. From $\theta_{S}$ at $L_{1}^{*}$ the Frank-Kamenetskii critical delta

$$
\delta_{s}=\frac{L^{* 2}}{\theta_{s}^{2} \exp \left(1 / \theta_{s}\right)}
$$

can be determined.

Tables 4 through 6 give $\theta_{\mathrm{m}}, \theta_{\mathrm{s}}$, and $\delta_{\mathrm{s}}$ at the critical condition for thermal explosion as function of $\theta_{\mathrm{m}}$ for the slab, cylinder, and sphere, respectively. 
The speed of convergence of the series solution varies with the geometry. As a consequence, 15 terms give very good agreement with the values given in Reference 4 for $\mathrm{k}=0$, for $\mathrm{k}=1$ there is some deterioration, and for $\mathrm{k}=2$ convergence is good for only a narrow range of $\theta$.

Equation (2) can be used to calculate the temperature profile in a material. Tables 4 through 6 show that the profile is very flat at the critical condition for small values of $\theta_{\mathrm{m}}$ and, conversely, the profile is steep for large values of $\theta_{\mathrm{m}}$. This illustrates the reason why the Frank-Kamenetskii exponential approximation gives good results for small values of $\theta_{s}$ (or $\theta_{m}$ ) but does not give good results for large values of $\theta_{s^{*}}$. That is, the exponential approximation is valid for only small departures from $\theta_{\mathbf{s}^{\circ}}$

\section{Conclusions}

The series solution method shown herein is a rather general and straightforward method which gives values in good agreement with other methods utilizing substantially more computer time and programming sophistication. The method appears to be well suited to investigating other problems involving nonlinear heat sources/sinks as well as variations of the thermal explosion problem, such as composites and heat flux or mixed boundary conditions. 
References

1. Semenov, N。 N., Z. Phys., $\underline{48}, 571$ (1928).

2. Frank-Kamenetskii, D. A., Diffusion and Heat Transfer in Chemical Kinetics 2nd ed., Plenum Press, 1969, pp. 460-506.

3. Chambré, P. L., J. Chem. Phys., 20, 1795 (1952)。

4. Enig, J. W., Shanks, D., and Southworth, R. W., The Numerical Solution of the Heat Conduction Equation Occurring in the Theory of Thermal Explosion, U.S. Naval Ordnance Laboratory, NAVORD 4377, November 1956.

5. Hardee, H. C., Lee, D. O., and Donaldson, A. B., Combustion and Flame, 18, 403 (1972).

6. Shouman, A. R., Donaldson, A. B., and Tsao, H. Y., Combustion and Flame, $\underline{23}, 17(1974)$ 。 
Table 1

The $a(1, n)^{\prime} s$ for Cartesian Geometry $(k=0)$

$$
\begin{aligned}
& a(1,1)=\left[5.00000 \times 10^{-1}\right] \exp \left(-1 / \theta_{m}\right) \\
& a(1,2)=\left[-4.16667 \times 10^{-2} \theta_{m}^{-2}\right] \exp \left(-2 / \theta_{m}\right) \\
& a(1,3)=\left[-8.33333 \times 10^{-3} \theta_{m}^{-3}+5.55555 \times 10^{-3} \theta_{m}^{-4}\right] \exp \left(-3 / \theta_{m}\right) \\
& a(1,4)=\left[-2.23214 \times 10^{-3} \theta_{m}^{-4}+3.12500 \times 10^{-3} \theta_{m}^{-5}\right. \\
& \left.-8.43254 \times 10^{-4} \theta_{m}^{-6}\right] \exp \left(-4 / \theta_{m}\right) \\
& a(1,5)=\left[-6.94444 \times 10^{-4} \theta_{m}^{-5}+1.50628 \times 10^{-3} \theta_{m}^{-6}\right. \\
& \left.-8.56481 \times 10^{-4} \theta_{m}^{-7}+1.36684 \times 10^{-4} \theta_{m}^{-8}\right] \exp \left(-5 / \theta_{m}\right) \\
& a(1,6)=\left[-2.36742 \times 10^{-4} \theta_{m}^{-6}+7.00833 \times 10^{-4} \theta_{m}^{-7}\right. \\
& -6.20929 \times 10^{-4} \theta_{m}^{-8}+2.08408 \times 10^{-4} \theta_{m}^{-9} \\
& \left.-2.30813 \times 10^{-5} \theta_{m}^{-10}\right] \exp \left(6 / \theta_{m}\right) \\
& a(1,7)=\left[-8.58516 \times 10^{-5} \theta_{m}^{-7}+3.23381 \times 10^{-4} \theta_{m}^{-8}\right. \\
& -3.93322 \times 10^{-4} \theta_{m}^{-9}+2.05838 \times 10^{-4} \theta_{m}^{-10} \\
& \left.-4.78033 \times 10^{-5} \theta_{m}^{-11}+4.00907 \times 10^{-6} \theta_{m}^{-12}\right] \exp \left(-7 / \theta_{m}\right)
\end{aligned}
$$


Table 1 (cont.)

$$
\begin{aligned}
& a(1,8)=\left[-3.25521 \times 10^{-5} \theta_{m}^{-8}+1.49224 \times 10^{-4} \theta_{m}^{-9}\right. \\
& -2.32195 \times 10^{-4} \theta_{\mathrm{m}}^{-10}+1.67094 \times 10^{-4} \theta_{\mathrm{m}}^{-11} \\
& -6.04517 \times 10^{-5} \theta_{m}^{-12}+1.05995 \times 10^{-5} \theta_{m}^{-13} \\
& \left.-7.10857 \times 10^{-7}\right] \exp \left(-8 / \theta_{m}\right) \\
& \mathrm{a}(1,9)=\left[-1.27655 \times 10^{-5} \theta_{\mathrm{m}}^{-9}+6.90601 \times 10^{-5} \theta_{\mathrm{m}}^{-10}\right. \\
& -1.31417 \times 10^{-4} \theta_{m}^{-11}+1.21234 \times 10^{-4} \theta_{m}^{-12} \\
& -6.03207 \times 10^{-5} \theta_{\mathrm{m}}^{-13}+1.64531 \times 10^{-4} \theta_{\mathrm{m}}^{-14} \\
& \left.-2.30057 \times 10^{-6} \theta_{m}^{-15}+1.28044 \times 10^{-7} \theta_{m}^{-16}\right] \exp \left(-9 / \theta_{m}\right) \\
& \mathrm{a}(1,10)=\left[-5.13980 \times 10^{-6} \theta_{\mathrm{m}}^{-10}+3.20835 \times 10^{-5} \theta_{\mathrm{m}}^{-11}\right. \\
& -7.23646 \times 10^{-5} \theta_{m}^{-12}+8.18138 \times 10^{-5} \theta_{m}^{-13} \\
& -5.22184 \times 10^{-5} \theta_{\mathrm{m}}^{-14}+1.95788 \times 10^{-5} \theta_{\mathrm{m}}^{-15} \\
& -4.25218 \times 10^{-6} \theta_{\mathrm{m}}^{-16}+4.92197 \times 10^{-7} \theta_{\mathrm{m}}^{-17} \\
& \left.-2.33525 \times 10^{-8} \theta_{m}^{-18}\right] \exp \left(-10 / \theta_{m}\right)
\end{aligned}
$$




\section{Table 1 (cont.)}

$$
\begin{aligned}
\mathrm{a}(1,11)= & {\left[-2.11377 \times 10^{-6} \theta_{\mathrm{m}}^{-11}+1.49649 \times 10^{-5} \theta_{\mathrm{m}}^{-12}\right.} \\
& -3.90974 \times 10^{-5} \theta_{\mathrm{m}}^{-13}+5.25004 \times 10^{-5} \theta_{\mathrm{m}}^{-14} \\
& -4.11049 \times 10^{-5} \theta_{\mathrm{m}}^{-15}+1.97709 \times 10^{-5} \theta_{\mathrm{m}}^{-16} \\
& -5.89786 \times 10^{-6} \theta_{\mathrm{m}}^{-17}+1.05876 \times 10^{-6} \theta_{\mathrm{m}}^{-18} \\
& \left.-1.04236 \times 10^{-7} \theta_{\mathrm{m}}^{-19}+4.30110 \times 10^{-9} \theta_{\mathrm{m}}^{-20}\right] \exp \left(-11 / \theta_{\mathrm{m}}\right)
\end{aligned}
$$

$$
\begin{aligned}
a(1,12)= & -8.84567 \times 10^{-7} \theta_{\mathrm{m}}^{-12}+7.00718 \times 10^{-6} \theta_{\mathrm{m}}^{-13} \\
& -2.08353 \times 10^{-5} \theta_{\mathrm{m}}^{-14}+3.02273 \times 10^{-5} \theta_{\mathrm{m}}^{-15} \\
& -3.02274 \times 10^{-5} \theta_{\mathrm{m}}^{-16}+1.78409 \times 10^{-5} \theta_{\mathrm{m}}^{-17} \\
& -6.82540 \times 10^{-6} \theta_{\mathrm{m}}^{-18}+1.68143 \times 10^{-6} \theta_{\mathrm{m}}^{-19} \\
& -2.56352 \times 10^{-7} \theta_{\mathrm{m}}^{-20}+2.19101 \times 10^{-8} \theta_{\mathrm{m}}^{-21} \\
& \left.-7.99120 \times 10^{-10} \theta_{\mathrm{m}}^{-22}\right] \exp \left(-12 / \theta_{\mathrm{m}}\right)
\end{aligned}
$$

$$
\begin{aligned}
a(1,13)= & {\left[-3.75601 \times 10^{-7} \theta_{\mathrm{m}}^{-13}+3.29292 \times 10^{-6} \theta_{\mathrm{m}}^{-14}\right.} \\
& -1.09896 \times 10^{-5} \theta_{\mathrm{m}}^{-15}+1.95281 \times 10^{-5} \theta_{\mathrm{m}}^{-16} \\
& -2.11213 \times 10^{-5} \theta_{\mathrm{m}}^{-17}+1.48230 \times 10^{-5} \theta_{\mathrm{m}}^{-18} \\
& -6.96209 \times 10^{-6} \theta_{\mathrm{m}}^{-19}+2.19850 \times 10^{-6} \theta_{\mathrm{m}}^{-20} \\
& -4.59542 \times 10^{-7} \theta_{\mathrm{m}}^{-21}+6.07385 \times 10^{-8} \theta_{\mathrm{m}}^{22} \\
& \left.-4.57933 \times 10^{-9} \theta_{\mathrm{m}}^{-23}+1.49479 \times 10^{-10} \theta_{\mathrm{m}}^{-24}\right] \exp \left(-13 / \theta_{\mathrm{m}}\right)
\end{aligned}
$$


Table 1 (cont.)

$$
\begin{aligned}
\mathrm{a}(1,14)= & {\left[-1.61469 \times 10^{-7} \theta_{\mathrm{m}}^{-14}+1.55262 \times 10^{-6} \theta_{\mathrm{m}}^{-15}\right.} \\
& -5.75077 \times 10^{-6} \theta_{\mathrm{m}}^{-16}+1.14891 \times 10^{-5} \theta_{\mathrm{m}}^{-17} \\
& -1.41841 \times 10^{-5} \theta_{\mathrm{m}}^{-18}+1.15785 \times 10^{-5} \theta_{\mathrm{m}}^{-19} \\
& -6.46762 \times 10^{-6} \theta_{\mathrm{m}}^{-20}+2.50576 \times 10^{-6} \theta_{\mathrm{m}}^{-21} \\
& -6.71001 \times 10^{-7} \theta_{\mathrm{m}}^{-22}+1.21476 \times 10^{-7} \theta_{\mathrm{m}}^{-23} \\
& -1.41448 \times 10^{-8} \theta_{\mathrm{m}}^{-24}+9.52882 \times 10^{-10} \theta_{\mathrm{m}}^{-25} \\
& \left.-2.81271 \times 10^{-11} \theta_{\mathrm{m}}^{-26}\right] \exp \left(-14 / \theta_{\mathrm{m}}\right)
\end{aligned}
$$

$$
\begin{aligned}
\mathrm{a}(1,15)= & {\left[-7.01554 \times 10^{-8} \theta_{\mathrm{m}}^{-15}+7.34307 \times 10^{-7} \theta_{\mathrm{m}}^{-16}\right.} \\
& -2.99061 \times 10^{-6} \theta_{\mathrm{m}}^{-17}+6.64149 \times 10^{-6} \theta_{\mathrm{m}}^{-18} \\
& -9.22841 \times 10^{-6} \theta_{\mathrm{m}}^{-19}+8.60448 \times 10^{-6} \theta_{\mathrm{m}}^{-20} \\
& -5.58978 \times 10^{-6} \theta_{\mathrm{m}}^{-21}+2.57683 \times 10^{-6} \theta_{\mathrm{m}}^{-22} \\
& -8.46332 \times 10^{-7} \theta_{\mathrm{m}}^{-23}+1.96166 \times 10^{-7} \theta_{\mathrm{m}}^{-24} \\
& -3.12578 \times 10^{-8} \theta_{\mathrm{m}}^{-25}+3.24820 \times 10^{-9} \theta_{\mathrm{m}}^{-26} \\
& \left.-1.97584 \times 10^{-10} \theta_{\mathrm{m}}^{-27}+5.31977 \times 10^{-12} \theta_{\mathrm{m}}^{-28}\right] \exp \left(-15 / \theta_{\mathrm{m}}\right)
\end{aligned}
$$




\section{Table 2}

The $a(1, n)$ 's for Cylindrical Geometry $(k=1)$

$$
\begin{aligned}
& \mathrm{a}(1,1)= {\left[2.50000 \times 10^{-1}\right] \exp \left(-1 / \theta_{\mathrm{m}}\right) } \\
& \mathrm{a}(1,2)= {\left[1.56250 \times 10^{-2} \theta_{\mathrm{m}}^{-2}\right] \exp \left(-2 / \theta_{\mathrm{m}}\right) } \\
& \mathrm{a}(1,3)= {\left[-1.73611 \times 10^{-3} \theta_{\mathrm{m}}^{-3}+1.30208 \times 10^{-3} \theta_{\mathrm{m}}^{-4}\right] \exp \left(-3 / \theta_{\mathrm{m}}\right) } \\
& \mathrm{a}(1,4)= {\left[-2.44141 \times 10^{-4} \theta_{\mathrm{m}}^{-4}+3.03338 \times 10^{-4} \theta_{\mathrm{m}}^{-5}\right.} \\
&\left.-1.22070 \times 10^{-4} \theta_{\mathrm{m}}^{-6}\right] \exp \left(-4 / \theta_{\mathrm{m}}\right) \\
& \mathrm{a}(1,5)= {\left[-3.90625 \times 10^{-5} \theta_{\mathrm{m}}^{-5}+9.90126 \times 10^{-5} \theta_{\mathrm{m}}^{-6}\right.} \\
&\left.-6.60536 \times 10^{-5} \theta_{\mathrm{m}}^{-7}+1.22070 \times 10^{-5} \theta_{\mathrm{m}}^{-8}\right] \exp \left(-5 / \theta_{\mathrm{m}}\right) \\
&-8.19798 \times 10^{-6} \theta_{\mathrm{m}}^{-9}+5.12651 \times 10^{-6} \theta_{\mathrm{m}}^{-10} \\
&\left.-1.40179 \times 10^{-6} \theta_{\mathrm{m}}^{-11}+1.36239 \times 10^{-7} \theta_{\mathrm{m}}^{-12}\right] \exp \left(-7 / \theta_{\mathrm{m}}\right) \\
& \mathrm{a}(1,7)= {\left[-1.24562 \times 10^{-6} \theta_{\mathrm{m}}^{-7}+5.59247 \times 10^{-6} \theta_{\mathrm{m}}^{-8}\right.} \\
&-2.50357 \times 10^{-5} \theta_{\mathrm{m}}^{-8}+9.90126 \times 10^{-6} \theta_{\mathrm{m}}^{-9} \\
& \mathrm{a}(1,6)= {\left[-6.78168 \times 10^{-6} \theta_{\mathrm{m}}^{-6}+2.37246 \times 10^{-5} \theta_{\mathrm{m}}^{-7}\right.} \\
&\left.\theta^{-10}\right] \exp \left(-6 / \theta_{\mathrm{m}}\right)^{\prime}
\end{aligned}
$$




\section{Table 2 (cont.)}

$$
\begin{aligned}
& a(1,8)=\left[-2.38419 \times 10^{-7} \theta_{m}^{-8}+1.31166 \times 10^{-6} \theta_{m}^{-9}\right. \\
& -2.48348 \times 10^{-6} \theta_{m}^{-10}+2.16036 \times 10^{-6} \theta_{m}^{-11} \\
& -9.32120 \times 10^{-7} \theta_{\mathrm{m}}^{-12}+1.91980 \times 10^{-7} \theta_{\mathrm{m}}^{-13} \\
& \left.-1.49012 \times 10^{-8} \theta_{m}^{-14}\right] \exp \left(-8 / \theta_{m}\right) \\
& \mathrm{a}(1,9)=\left[-4.70950 \times 10^{-8} \theta_{\mathrm{m}}^{-9}+3.07481 \times 10^{-7} \theta_{\mathrm{m}}^{-10}\right. \\
& -7.17642 \times 10^{-7} \theta_{\mathrm{m}}^{-11}+8.08084 \times 10^{-7} \theta_{\mathrm{m}}^{-12} \\
& -4.84784 \times 10^{-7} \theta_{\mathrm{m}}^{-13}+1.57152 \times 10^{-7} \theta_{\mathrm{m}}^{-14} \\
& \left.-2.57416 \times 10^{-8} \theta_{m}^{-15}+1.65568 \times 10^{-9} \theta_{m}^{-16}\right] \exp \left(-9 / \theta_{m}\right) \\
& a(1,10)=\left[-9.53674 \times 10^{-9} \theta_{m}^{-10}+7.21808 \times 10^{-8} \theta_{m}^{-11}\right. \\
& -2.01017 \times 10^{-7} \theta_{m}^{-12}+2.79715 \times 10^{-7} \theta_{m}^{-13} \\
& -2.17306 \times 10^{-7} \theta_{\mathrm{m}}^{-14}+9.78279 \times 10^{-8} \theta_{\mathrm{m}}^{-15} \\
& -2.51571 \times 10^{-8} \theta_{m}^{-16}+3.40222 \times 10^{-9} \theta_{m}^{-17} \\
& \left.-1.86245 \times 10^{-10} \theta_{m}^{-18}\right] \exp \left(-10 / \theta_{m}\right)
\end{aligned}
$$




\section{Table 2 (cont.)}

$$
\begin{aligned}
\mathrm{a}(1,11)= & -1.97040 \times 10^{-9} \theta_{\mathrm{m}}^{-11}+1.69819 \times 10^{-8} \theta_{\mathrm{m}}^{-12} \\
& -5.50949 \times 10^{-8} \theta_{\mathrm{m}}^{-13}+9.17128 \times 10^{-8} \theta_{\mathrm{m}}^{-14} \\
& -8.81281 \times 10^{-8} \theta_{\mathrm{m}}^{-15}+5.13564 \times 10^{-8} \theta_{\mathrm{m}}^{-16} \\
& -1.83131 \times 10^{-8} \theta_{\mathrm{m}}^{-17}+3.87880 \times 10^{-9} \theta_{\mathrm{m}}^{-18} \\
& \left.-4.45073 \times 10^{-10} \theta_{\mathrm{m}}^{-19}+2.11664 \times 10^{-11} \theta_{\mathrm{m}}^{-20}\right] \exp \left(-11 / \theta_{\mathrm{m}}\right)
\end{aligned}
$$

$$
\begin{aligned}
& a(1,12)=\left[-4.139211 \times 10^{-10} \theta_{\mathrm{m}}^{-12}+4.00531 \times 10^{-9} \theta_{\mathrm{m}}^{-13}\right. \\
& -1.48628 \times 10^{-8} \theta_{\mathrm{m}}^{-14}+2.88957 \times 10^{-8} \theta_{\mathrm{m}}^{-15} \\
& -3.32555 \times 10^{-8} \theta_{\mathrm{m}}^{-16}+2.39726 \times 10^{-8} \theta_{\mathrm{m}}^{-17} \\
& -1.10567 \times 10^{-8} \theta_{\mathrm{m}}^{-18}+3.24227 \times 10^{-9} \theta_{\mathrm{m}}^{-19} \\
& -5.81359 \times 10^{-10} \theta_{m}^{-20}+5.77830 \times 10^{-11} \theta_{m}^{-21} \\
& \left.-2.42532 \times 10^{-12} \theta_{m}^{-22}\right] \exp \left(-12 / \theta_{m}\right) \\
& a(1,13)=\left[-8.81725 \times 10^{-11} \theta_{m}^{-13}+9.47104 \times 10^{-10} \cdot \theta_{m}^{-14}\right. \\
& -3.96186 \times 10^{-9} \theta_{m}^{-15}+8.83132 \times 10^{-9} \theta_{m}^{-16} \\
& -1.18861 \times 10^{-8} \theta_{\mathrm{m}}^{-17}+1.02664 \times 10^{-8} \theta_{\mathrm{m}}^{-18} \\
& -5.85533 \times 10^{-9} \theta_{m}^{-19}+2.21866 \times 10^{-9} \theta_{m}^{-20} \\
& -5.49925 \times 10^{-10} \theta_{\mathrm{m}}^{-21}+8.52375 \times 10^{-11} \theta_{\mathrm{m}}^{-22} \\
& \left.-7.45853 \times 10^{-12} \theta_{m}^{-23}+2.79845 \times 10^{-13} \theta_{m}^{-24}\right] \exp \left(-13 / \theta_{m}\right)
\end{aligned}
$$




\section{Table 2 (cont.)}

$$
\begin{aligned}
& a(1,14)=\left[-1.90066 \times 10^{-11} \theta_{m}^{-14}+2.24513 \times 10^{-10} \theta_{m}^{-15}\right. \\
& -1.04635 \times 10^{-9} \theta_{m}^{-16}+2.63535 \times 10^{-9} \theta_{m}^{-17} \\
& -4.07229 \times 10^{-9} \theta_{\mathrm{m}}^{-18}+4.11593 \times 10^{-9} \theta_{\mathrm{m}}^{-19} \\
& -2.81270 \times 10^{-9} \theta_{m}^{-20}+1.31717 \times 10^{-9} \theta_{m}^{-21} \\
& -4.21417 \times 10^{-10} \theta_{\mathrm{m}}^{-21}+9.01592 \times 10^{-11} \theta_{\mathrm{m}}^{-23} \\
& -1.22798 \times 10^{-11} \theta_{m}^{-23}+9.58389 \times 10^{-13} \theta_{m}^{-25} \\
& \left.-3.24820 \times 10^{-14} \theta_{m}^{-25}\right] \exp \left(-14 / \theta_{m}\right) \\
& a(1,15)=\left[-4.13921 \times 10^{-12} \theta_{\mathrm{m}}^{-15}+5.33477 \times 10^{-11} \theta_{\mathrm{m}}^{-16}\right. \\
& -2.74326 \times 10^{-10} \theta_{\mathrm{m}}^{-17}+7.71444 \times 10^{-10} \theta_{\mathrm{m}}^{-18} \\
& -1.34878 \times 10^{-9} \theta_{m}^{-19}+1.56631 \times 10^{-9} \theta_{m}^{-20} \\
& -1.25277 \times 10^{-9} \theta_{\mathrm{m}}^{-21}+7.02736 \times 10^{-10} \theta_{\mathrm{m}}^{-22} \\
& -2.77677 \times 10^{-10} \theta_{m}^{-23}+7.66008 \times 10^{-11} \theta_{m}^{-24} \\
& -1.43806 \times 10^{-11} \theta_{\mathrm{m}}^{-25}+1.74398 \times 10^{-12} \theta_{\mathrm{m}}^{-26} \\
& \left.-1.22706 \times 10^{-13} \theta_{m}^{-27}+3.78956 \times 10^{-15} \theta_{m}^{-28}\right] \exp \left(-15 / \theta_{m}\right)
\end{aligned}
$$




\section{Table 3}

The $a(1, n)$ 's for Spherical Geometry $(k=2)$

$$
\begin{aligned}
& a(1,1)=\left[1.66667 \times 10^{-1}\right] \exp \left(-1 / \theta_{m}\right) \\
& a(1,2)=\left[8.33333 \times 10^{-3} \theta_{m}^{-2}\right] \exp \left(-2 / \theta_{m}\right) \\
& a(1,3)=\left[-6.61376 \times 10^{-4} \theta_{m}^{-3}+5.29101 \times 10^{-4} \theta_{m}^{-4}\right] \exp \left(-3 / \theta_{m}\right) \\
& a(1,4)=\left[-6.43004 \times 10^{-5} \theta_{m}^{-4}+1.12066 \times 10^{-4} \theta_{m}^{-5}\right. \\
& \left.-3.73554 \times 10^{-5} \theta_{m}^{-6}\right] \exp \left(-4 / \theta_{m}\right) \\
& a(1,5)=\left[-7.01459 \times 10^{-6} \theta_{m}^{-5}+1.94237^{-5} \theta_{m}^{-6}\right. \\
& \left.-1.40759 \times 10^{-5} \theta_{m}^{-7}+2.80138^{-6} \theta_{m}^{-8}\right] \exp \left(-5 / \theta_{m}\right) \\
& a(1,6)=\left[-8.24364 \times 10^{-7} \theta_{m}^{-6}+3.17362 \times 10^{-6} \theta_{m}^{-7}\right. \\
& -3.67006 \times 10^{-6} \theta_{m}^{-8}+1.57775 \times 10^{-6} \theta_{m}^{-9} \\
& \left.-2.18422 \times 10^{-7} \theta_{m}^{-10}\right] \exp \left(-6 / \theta_{m}\right) \\
& a(1,7)=\left[-1.02064 \times 10^{-7} \theta_{m}^{-7}+5.07155 \times 10^{-7} \theta_{m}^{-8}\right. \\
& -8.20484 \times 10^{-7} \theta_{\mathrm{m}}^{-9}+5.62042 \times 10^{-7} \theta_{\mathrm{m}}^{-10} \\
& \left.-1.66989 \times 10^{-7} \theta_{m}^{-11}+1.74996 \times 10^{-8} \theta_{m}^{-12}\right] \exp \left(-7 / \theta_{m}\right)
\end{aligned}
$$




\section{Table 3 (cont.)}

$$
\begin{aligned}
& a(1,8)=\left[-1.31333 \times 10^{-8} \theta_{m}^{-8}+8.03328 \times 10^{-8} \theta_{m}^{-9}\right. \\
& -1.68845 \times 10^{-7} \theta_{\mathrm{m}}^{-10}+1.61942 \times 10^{-7} \theta_{\mathrm{m}}^{-11} \\
& -7.64389 \times 10^{-8} \theta_{m}^{-12}+1.70931 \times 10^{-8} \theta_{m}^{-13} \\
& \left.-1.43045 \times 10^{-9} \theta_{\mathrm{m}}^{-14}\right] \exp \left(-8 / \theta_{\mathrm{m}}\right) \\
& a(1,9)=\left[-1.7486 \times 10^{-9} \theta_{m}^{-9}+1.26849 \times 10^{-8} \theta_{m}^{-10}\right. \\
& -3.30265 \times 10^{-8} \theta_{m}^{-11}+4.12317 \times 10^{-8} \theta_{m}^{-12} \\
& -2.72206 \times 10^{-8} \theta_{m}^{-13}+9.63883 \times 10^{-9} \theta_{m}^{-14} \\
& \left.-1.71270 \times 10^{-9} \theta_{m}^{-15}+1.18743 \times 10^{-10} \theta_{m}^{-16}\right] \exp \left(-9 / \theta_{m}\right) \\
& a(1,10)=\left[-2.36260 \times 10^{-10} \theta_{m}^{-10}+2.00205 \times 10^{-9} \theta_{m}^{-11}\right. \\
& -6.24581 \times 10^{-9} \theta_{\mathrm{m}}^{-12}+9.68248 \times 10^{-9} \theta_{\mathrm{m}}^{-13} \\
& -8.82090 \times 10^{-9} \theta_{\mathrm{m}}^{-14}+4.11386 \times 10^{-9} \theta_{\mathrm{m}}^{-15} \\
& -1.15388 \times 10^{-9} \theta_{\mathrm{m}}^{-16}+1.69133 \times 10^{-10} \theta_{\mathrm{m}}^{-17} \\
& \left.-9.97801 \times 10^{-12} \theta_{m}^{-18}\right] \exp \left(-10 / \theta_{m}\right)
\end{aligned}
$$




\section{Table 3 (cont.)}

$$
\begin{aligned}
& a(1,11)=\left[-3.26841 \times 10^{-11} \theta_{\mathrm{m}}^{-11}+3.16240 \times 10^{-10} \theta_{\mathrm{m}}^{-12}\right. \\
& -1.15350 \times 10^{-9} \theta_{\mathrm{m}}^{-13}+2.14827 \times 10^{-9} \theta_{\mathrm{m}}^{-14} \\
& -2.29402 \times 10^{-9} \theta_{\mathrm{m}}^{-15}+1.47520 \times 10^{-9} \theta_{\mathrm{m}}^{-16} \\
& -5.76590 \times 10^{-10} \theta_{m}^{-17}+1.33022 \times 10^{-10} \theta_{m}^{-18} \\
& \left.-1.65298 \times 10^{-11} \theta_{m}^{-19}+8.46795 \times 10^{-13} \theta_{m}^{-20}\right] \exp \left(-11 / \theta_{m}\right) \\
& a(1,12)=\left[-4.59394 \times 10^{-12} \theta_{\mathrm{m}}^{-12}+5.00239 \times 10^{-11} \theta_{\mathrm{m}}^{-13}\right. \\
& -2.09363 \times 10^{-10} \theta_{m}^{-14}+4.57097 \times 10^{-10} \theta_{m}^{-15} \\
& -5.87012 \times 10^{-10} \theta_{\mathrm{m}}^{-16}+4.68978 \times 10^{-10} \theta_{\mathrm{m}}^{-17} \\
& -2.38145 \times 10^{-10} \theta_{\mathrm{m}}^{-18}+7.64101 \times 10^{-11} \theta_{\mathrm{m}}^{-19} \\
& -1.49050 \times 10^{-11} \theta_{m}^{-20}+1.60311 \times 10^{-12} \theta_{m}^{-21} \\
& \left.-7.24555 \times 10^{-14} \theta_{m}^{-22}\right] \exp \left(-12 / \theta_{m}\right) \\
& a(1,13)=\left[-6.54407 \times 10^{-13} \theta_{m}^{-13}+7.92659 \times 10^{-12} \theta_{m}^{-14}\right. \\
& -3.75028 \times 10^{-11} \theta_{m}^{-15}+9.41928 \times 10^{-11} \theta_{m}^{-16} \\
& -1.41985 \times 10^{-10} \theta_{m}^{-17}+1.36452 \times 10^{-10} \theta_{m}^{-18} \\
& -8.60316 \times 10^{-11} \theta_{\mathrm{m}}^{-19}+3.58161 \times 10^{-11} \theta_{\mathrm{m}}^{-20} \\
& -9.69815 \times 10^{-12} \theta_{m}^{-21}+1.63349 \times 10^{-12} \theta_{m}^{-22} \\
& \left.-1.54564 \times 10^{-13} \theta_{m}^{-23}+6.24250 \times 10^{-15} \theta_{m}^{-24}\right] \exp \left(-13 / \theta_{m}\right)
\end{aligned}
$$


Table 3 (cont.)

$$
\begin{aligned}
& a(1,14)=\left[-9.42926 \times 10^{-14} \theta_{\mathrm{m}}^{-14}+1.25831 \times 10^{-12} \theta_{\mathrm{m}}^{-15}\right. \\
& -6.64940 \times 10^{-12} \theta_{\mathrm{m}}^{-16}+1.89268 \times 10^{-11} \theta_{\mathrm{m}}^{-17} \\
& -3.28659 \times 10^{-11} \theta_{\mathrm{m}}^{-18}+3.70923 \times 10^{-11} \theta_{\mathrm{m}}^{-19} \\
& -2.81253 \times 10^{-11} \theta_{\mathrm{m}}^{-20}+1.45261 \times 10^{-11} \theta_{\mathrm{m}}^{-21} \\
& -5.09674 \times 10^{-12} \theta_{m}^{-22}+1.18953 \times 10^{-12} \theta_{m}^{-23} \\
& -1.75882 \times 10^{-13} \theta_{\mathrm{m}}^{-24}+1.48340 \times 10^{-14} \theta_{\mathrm{m}}^{-25} \\
& \left.-5.41002 \times 10^{-16} \theta_{m}^{-26}\right] \exp \left(-14 / \theta_{m}\right) \\
& a(1,15)=\left[-1.37214 \times 10^{-14} \theta_{\mathrm{m}}^{-15}+2.00115 \times 10^{-13} \theta_{\mathrm{m}}^{-16}\right. \\
& -1.16942 \times 10^{-12} \theta_{\mathrm{m}}^{-17}+3.72665 \times 10^{-12} \theta_{\mathrm{m}}^{-18} \\
& -7.34422 \times 10^{-12} \theta_{\mathrm{m}}^{-19}+9.55468 \times 10^{-12} \theta_{\mathrm{m}}^{-20} \\
& -8.50849 \times 10^{-12} \theta_{\mathrm{m}}^{-21}+5.28242 \times 10^{-12} \theta_{\mathrm{m}}^{-22} \\
& -2.29724 \times 10^{-12} \theta_{\mathrm{m}}^{-23}+6.93829 \times 10^{-13} \theta_{\mathrm{m}}^{-24} \\
& -1.41917 \times 10^{-13} \theta_{\mathrm{m}}^{-25}+1.86668 \times 10^{-14} \theta_{\mathrm{m}}^{-26} \\
& \left.-1.41847 \times 10^{-15} \theta_{m}^{-26}+4.71240 \times 10^{-17} \theta_{m}^{-28}\right] \exp \left(-15 / \theta_{m}\right)
\end{aligned}
$$


Table 4

Critical Parameters for Cartesian Geometry $(k=0)$

\begin{tabular}{|c|c|c|c|c|}
\hline${ }_{\mathrm{m}}$ & $\theta_{\mathrm{s}}^{*}$ & $\delta_{\mathrm{m}}^{*}$ & $\delta_{\mathrm{m}}^{*}$ (Ref.4) & Rel. Dev \\
\hline 0.50000 & 0.24459 & 2.51405 & 2.51541 & 0.00054 \\
\hline 0.33333 & 0.21385 & 2.63346 & 2.63458 & 0.00043 \\
\hline 0.25000 & 0.18108 & 2.69402 & 2.69504 & 0.00038 \\
\hline 0.20000 & 0.15523 & 2.73060 & 2.73155 & 0.00035 \\
\hline 0.16667 & 0.13526 & 2.75508 & 2.75598 & 0.00033 \\
\hline 0.14286 & 0.11962 & 2.77261 & 2.77348 & 0.00031 \\
\hline 0.12500 & 0.10712 & 2.78577 & 2.78662 & 0.00030 \\
\hline 0.11111 & 0.09692 & 2.79603 & 2.79686 & 0.00030 \\
\hline 0.10000 & 0.08847 & 2.80424 & 2.80506 & 0.00029 \\
\hline 0.09090 & 0.08136 & 2.81097 & 2.81177 & 0.00029 \\
\hline 0.08333 & 0.07529 & 2.81657 & 2.81737 & 0.00028 \\
\hline 0.07692 & 0.07006 & 2.82132 & 2.82210 & 0.00028 \\
\hline 0.07143 & 0.06550 & 2.82539 & 2.82617 & 0.00028 \\
\hline 0.06667 & 0.06149 & 2.82892 & 2.82969 & 0.00027 \\
\hline 0.06250 & 0.05795 & 2.83201 & 2.83277 & 0.00027 \\
\hline 0.05882 & 0.05479 & 2.83474 & 2.83549 & 0.00027 \\
\hline 0.05556 & 0.05195 & 2.83716 & 2.83492 & 0.00027 \\
\hline 0.05263 & 0.04939 & 2.83933 & 2.84008 & 0.00026 \\
\hline 0.05000 & 0.04708 & 2.84128 & 2.84203 & 0.00026 \\
\hline 0.04762 & 0.04496 & 2.84305 & 2.84379 & 0.00026 \\
\hline
\end{tabular}

*Percentage deviation of $\delta_{m}^{*}$, by the series method, from the values in Reference 4. 
Table 4 (cont.)

\begin{tabular}{|c|c|c|c|c|}
\hline${ }_{\mathrm{m}}$ & $\theta_{\mathrm{s}}^{*}$ & $\delta_{\mathrm{m}}^{*}$ & $\delta_{m}^{*}$ (Ref.4) & Rel. Dev. \\
\hline 0.04545 & 0.04303 & 2.84466 & 2.84540 & 0.00026 \\
\hline 0.04348 & 0.04126 & 2.84612 & 2.84686 & 0.00026 \\
\hline 0.04167 & 0.03963 & 2.84747 & 2.84820 & 0.00026 \\
\hline 0.04000 & 0.03812 & 2.84871 & 2.84944 & 0.00026 \\
\hline 0.03846 & 0.03673 & 2.84985 & 2.85058 & 0.00026 \\
\hline 0.03704 & 0.03543 & 2.85090 & 2.85163 & 0.00025 \\
\hline 0.03571 & 0.03422 & 2.85189 & 2.85262 & 0.00025 \\
\hline 0.03448 & 0.03309 & 2.85281 & 2.85353 & 0.00025 \\
\hline 0.03333 & 0.03203 & 2.85366 & 2.85438 & 0.00025 \\
\hline 0.03226 & 0.03103 & 2.85443 & 2.85518 & 0.00026 \\
\hline 0.03125 & 0.03010 & 2.85519 & 2.85592 & 0.00026 \\
\hline 0.03030 & 0.02922 & 2.85593 & 2.85663 & 0.00024 \\
\hline 0.02941 & 0.02839 & 2.85657 & 2.85729 & 0.00025 \\
\hline 0.02857 & 0.02761 & 2.85720 & 2.85791 & 0.00025 \\
\hline 0.02778 & 0.02687 & 2.85780 & 2.85850 & 0.00024 \\
\hline 0.02703 & 0.02617 & 2.85835 & 2.85905 & 0.00025 \\
\hline 0.02632 & 0.02550 & 2.85888 & 2.85958 & 0.00025 \\
\hline 0.02564 & 0.02487 & 2.85938 & 2.86008 & 0.00025 \\
\hline 0.02500 & 0.02426 & 2.85985 & 2.86056 & 0.00025 \\
\hline 0.02439 & 0.02369 & 2.86031 & 2.86101 & 0.00025 \\
\hline 0.02381 & 0.02314 & 2.86074 & 2.86144 & 0.00024 \\
\hline
\end{tabular}


Table 4 (conto)

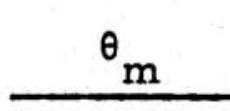

0.02326

0.02273

0.02222

0.02174

0.02128

0.02083

0.02041

0.02000

0.01961

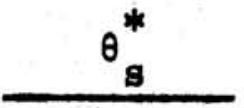

0.02262

0.02212

0.02164

0.02118

0.02074

0.02032

0.01998

0.01953

0.01915

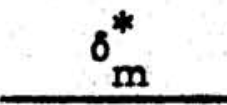

2.86114

2.86155

2.86192

2.86228

2.86262

2.86295

2.86327

2.86357

2.86387 $8_{\mathrm{m}}^{*}$ (Ref. 4)

2,86188

2.86224

2.86262

2.86287

2.86332

2.86365

2.86396

2.86426

2.86455
Rel. Dev.

0.00028

0.00024

0.00024

0.00024

0.00024

0.00024

0.00024

0.00024

0.00024 
Table 5

Critical Parameters for Cylindrical Geometry $(\mathrm{k}=1)$

\begin{tabular}{|c|c|c|c|c|}
\hline${ }^{\theta} \mathrm{m}$ & $\theta_{\mathbf{s}}^{*}$ & $\delta_{m}^{*}$ & $\delta_{\mathrm{m}}^{*}\left(\operatorname{Ref}_{0} 4\right)$ & Rel。Dev. \\
\hline 0.50000 & 0.23811 & 6.06110 & 6.06110 & 0.00000 \\
\hline 0.33333 & 0.20599 & 6.62980 & 6.62980 & 0.00000 \\
\hline 0.25000 & 0.17498 & 6.94143 & 6.94143 & 0.00000 \\
\hline 0.20000 & 0.15061 & 7.13780 & 7.13780 & 0.00000 \\
\hline 0.16667 & 0.13171 & 7.27280 & 7.27280 & 0.00000 \\
\hline 0.14286 & 0.11682 & 7.37128 & 7.37128 & 0.00000 \\
\hline 0.12500 & 0.10486 & 7.44631 & 7.44628 & 0.00000 \\
\hline 0.11111 & 0.09507 & 7.50554 & 7.50531 & 0.00003 \\
\hline 0.10000 & 0.08692 & 7.55387 & 7.55297 & 0.00012 \\
\hline 0.09091 & 0.08005 & 7.59470 & 7.59225 & 0.00032 \\
\hline 0.08333 & 0.07417 & 7.63093 & 7.62520 & 0.00175 \\
\hline 0.07692 & 0.06908 & 7.66605 & 7.65321 & 0.00168 \\
\hline 0.07143 & 0.06465 & 7.70643 & 7.67733 & 0.00379 \\
\hline 0.06667 & 0.06074 & 7.72520 & 7.69832 & 0.00349 \\
\hline 0.06250 & 0.05728 & 7.74821 & 7.71674 & 0.00408 \\
\hline 0.05882 & 0.05419 & 7.83124 & 7.73304 & 0.01270 \\
\hline 0.05556 & 0.05141 & 7.79371 & 7.74757 & 0.00596 \\
\hline 0.05263 & 0.04891 & 7.74963 & 7.76060 & 0.00141 \\
\hline 0.05000 & 0.04663 & 7.82419 & 7.77231 & 0.00668 \\
\hline 0.04762 & 0.04456 & 7.83430 & 7.78295 & 0.00660 \\
\hline
\end{tabular}


Table 5 (cont.)

\begin{tabular}{|c|c|c|c|c|}
\hline$\theta_{\mathrm{m}}$ & $\theta_{\mathrm{s}}^{*}$ & $\delta_{m}^{*}$ & $8_{\mathrm{m}}^{*}$ (Rer. 4) & Rel. Dev. \\
\hline 0.04545 & 0.04267 & 7.83292 & 7.79264 & 0.00517 \\
\hline 0.04348 & 0.04092 & 7.84010 & 7.80152 & 0.00485 \\
\hline 0.04167 & 0.03932 & 7.83999 & 7.80966 & 0.00388 \\
\hline 0.04000 & 0.03783 & 7.84650 & 7.81715 & 0.00376 \\
\hline 0.03846 & 0.03646 & 7.87979 & 7.82409 & 0.00712 \\
\hline 0.03704 & 0.03518 & 9.80751 & 7.83049 & 0.25248 \\
\hline 0.03571 & 0.03398 & 7.70073 & 7.83653 & 0.01733 \\
\hline 0.03448 & 0.03287 & 7.80954 & 7.84210 & 0.00415 \\
\hline 0.03333 & 0.03182 & 7.72565 & 7.84730 & 0.01550 \\
\hline 0.03226 & 0.03084 & 7.70350 & 7.85216 & 0.01893 \\
\hline 0.03125 & 0.02992 & 7.71305 & 7.85672 & 0.01829 \\
\hline 0.03030 & 0.02905 & 7.89245 & 7.86101 & 0.00400 \\
\hline 0.02941 & 0.02823 & 7.70762 & 7.86506 & 0.02002 \\
\hline 0.02857 & 0.02746 & 7.79010 & 7.86887 & 0.01001 \\
\hline 0.02778 & 0.02673 & 7.72857 & 7.87249 & 0.01828 \\
\hline 0.02703 & 0.02603 & 7.68923 & 7.87589 & 0.02370 \\
\hline 0.02632 & 0.02537 & 7.86617 & 7.87912 & 0.00165 \\
\hline 0.02564 & 0.02474 & 7.64780 & 7.88220 & 0.02974 \\
\hline 0.02500 & 0.02415 & 7.75915 & 7.88511 & 0.01597 \\
\hline 0.02439 & 0.02358 & 7.76185 & 7.88790 & 0.01598 \\
\hline 0.02381 & 0.02303 & 6.18600 & 7.89054 & 0.21602 \\
\hline
\end{tabular}


Table 5 (cont.)

\begin{tabular}{|c|c|c|c|c|}
\hline${ }_{\mathrm{m}}^{\theta}$ & $\theta_{\mathrm{s}}^{*}$ & $\delta_{m}$ & $\delta_{\mathrm{m}}^{*}$ (Ref. 4) & Rel. Dev. \\
\hline 0.02326 & 0.02252 & 7.84328 & 7.89305 & 0.00631 \\
\hline 0.02273 & 0.02202 & 7.68725 & 7.89547 & 0.02637 \\
\hline 0.02222 & 0.02155 & 7.69918 & 7.89778 & 0.02515 \\
\hline 0.02174 & 0.02109 & 7.53898 & 7.89998 & 0.04570 \\
\hline 0.02128 & 0.02066 & 7.79101 & 7.90209 & 0.01406 \\
\hline 0.02083 & 0.02024 & 7.51608 & 7.90411 & 0.04910 \\
\hline 0.02041 & 0.01984 & 7.59357 & 7.90606 & 0.03953 \\
\hline 0.02000 & 0.01945 & 8.00098 & 7.90791 & 0.01177 \\
\hline 0.01961 & 0.01908 & 7.59072 & 7.90971 & 0.04033 \\
\hline
\end{tabular}




\section{Table 6}

Critical Parameters for Spherical Geometry $(k=2)$

\begin{tabular}{l}
${ }^{\theta} \mathrm{m}$ \\
\hline 0.50000 \\
0.33333 \\
0.25000 \\
0.20000 \\
0.16667 \\
0.14286 \\
0.12500 \\
0.11111 \\
0.10000 \\
0.09091 \\
0.08333 \\
0.07692 \\
0.07143 \\
0.06667 \\
0.06250 \\
0.05882
\end{tabular}

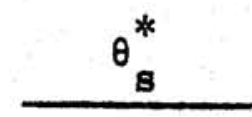

0.23103

0.19839

0.16900

0.14602

0.12813

0.11397

0.10254

0.09315

0.08531

0.07867

0.07298

0.06805

0.06374

0.05994

0.05657

0.05355

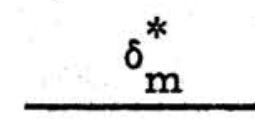

10.73846

12.25366

13.14741

13.73569

14.14855

14.53672

14.15495

13.37438

12.84802

12.46574

12.17404

11.94348

11.75634

11.60123

11.47051

11.36321 $\delta_{\mathrm{m}}^{*}$ (Ref. 4)

10.73919

12.25458

13.14846

13.73687

14.15324

14.46330

14.70309

14.89409

15.04974

15.17907

15.28823

15.38158

15.46230

15.53283

15.59496

15.65014
Rel.Devo

0.00007

0.00008

0.00008

0.00009

0.00033

0.00508

0.03728

0.10203

0.14630

0.17876

0.20370

0.22352

0.23968

0.25312

0.26447

0.27392 


\section{ISTRIBUTION:}

Los Alamos Scientific Laboratory (2)

P.O. Box 1663

Los Alamos, NM 87544

Attn: R. N. Rogers, WX-2, Mail Stop 920

A. Popolato, WX-3, Mail Stop 933

Lawrence Livermore Laboratory (2)

P.O. Box 808

Livermore, CA 94550

Attn: E. James, Mail Stop L 402

M. Finger, Mail Stop L 402

Mason and Hanger-Silas Mason, Inc.

Pantex Plant

P. O. Box 647

Amarillo, TX 79105

1283 H. C. Hardee

1283 D. O. Lee

1283 W. P. Schimmel, Jr.

2500 J. C. King

2510 D. H. Anderson

2513 D. B. Hayes

2513 A. B. Donaldson

2514 R. A. Damerow

2515 E. A. Kjeldgaard

2516 N. E. Brown

5131 L. W. Davison

5131 J. E. Kennedy

8115 D. Hartley

8344 A. R. Willis

9335 D. C. Bickel

9355 F. H. Mathews

8266 E. A. Aas (2)

3141 C. A. Pepmueller (Actg) (5)

3151 W. L. Garner (3)

For ERDA/TIC (Unlimited Release)

ERDA/TIC (25)

(R. P. Campbell, 3171-1) 